\title{
Modifying the electrical behaviour of polypropylene/carbon nanotube composites by adding a second nanoparticle and by annealing processes
}

\author{
H. Palza*, C. Garzón, O. Arias \\ Departamento de Ingeniería Química y Biotecnología, Facultad de Ciencias Físicas y Matemáticas, Universidad de Chile, \\ Beauchef 861, Casilla 277, Santiago, Chile
}

Received 28 December 2011; accepted in revised form 24 February 2012

\begin{abstract}
The effect of different nanoparticles on the geometrical percolation transition of multi-wall carbon nanotubes (CNT) in polypropylene (PP) composites was studied. Our results show that the electrical conductivity of PP/CNT composites (around $2 \mathrm{vol} \%$ ) can be tuned depending on the characteristic of the third component. Non-conductive layered silica fillers disrupt the CNT percolated network reducing the electrical conductivity of the composite. Spherical nanoparticles otherwise, either copper metal or silica-based, decrease the percolation threshold down to $0.5 \mathrm{vol} \%$ of CNT. These results cannot be explained by previous theories about the effect of a second particle on the electrical behaviour of polymer/CNT composites such as the interparticle bridging or the excluded volume. The effect of annealing in the melt was further analyzed and our results show that depending on the concentration and the type of filler, the electrical conductivity of the composites can be increased several orders of magnitude.
\end{abstract}

Keywords: nanocomposites, hybrid composite materials, metal nanoparticles, clay, carbon nanotubes

\section{Introduction}

The production of electrical conductive plastic materials seems to be one of the most important applications of polymer/carbon nanotube (CNT) composites in commodity materials such as polyolefins. The reason is the extremely low amount of filler needed to obtain the geometrical percolation threshold in these systems as compared with other nano or microfillers [1]. Motivated by the good results in this field, researchers have tried to decrease further the percolation transition by adding to polymer/CNT composites a third component, such as another polymer or a second particle [2-4]. A larger confinement of CNT has been reported as the driving force for the decrease in the percolation threshold in these hybrid composite materials $[2,3,5]$.
By adding clay particles into epoxy/single wall carbon nanotube (SWCNT) composites, Liu and Grunlan [4] found that the electrical conductivity percolation threshold of CNT can be reduced by a factor of five. SWCNTs appear to have an affinity for clay causing a more exfoliated and a better networked state in the composites explaining partially the results [4]. The excluded volume created by the micron-scale clay clusters forming a segregated network of nanotubes can also explain these conductivity improvements [4]. In PP/CNT composites, the addition of micro-particles of $\mathrm{CaCO}_{3}$ results in a significant reduction in the electrical resistivity that was explained by the concept of effective concentration similar to the excluded volume theory [5]. After incorporation of the inert micro-filler into the

\footnotetext{
${ }^{*}$ Corresponding author, e-mail: hpalza@ing.uchile.cl
}

(c) BME-PT 
composite, $\mathrm{CaCO}_{3}$ will hold up certain space increasing the effective concentration of CNT (volume percentage) and in this way the probability of connecting with each other. Similar results were reported by the same authors using other fillers (e.g. talc and wollastonite) and polymer matrices (e.g. polyoxymethylene and polyamide) [5]. This theory is further supported by the results from Kotaki et al. [6] in epoxy/clay/vapour grown carbon fiber hybrid composite materials. Despite the above mentioned results, a higher electrical percolation threshold in $\mathrm{PP} /$ clay/CNT hybrid composite materials than in $\mathrm{PP} / \mathrm{CNT}$ composites has been reported [7]. The disruption of the interconnecting conduction paths from the CNT because of the clay was stated as the reason for this drop in the conductivity.

An interesting novel hybrid composite material based on PP/CNT composites was developed by Liang et al. [8]. The addition of small amount of silver metal nanoparticles promoted significantly the electrical properties of the resulting material [8]. The mechanism proposed for this improvement was the anchoring of Ag particles among CNT clusters facilitating the electron transfer through the carbon filler. In this way, CNT act as bridges for individual silver particles, or vice versa, yielding high electrical conductivities and decreasing the percolation threshold. This model is supported by the results of Balik and coworkers [2,3] studying hybrid composite materials composed of blend matrices with carbon fiber and graphite particles. In particular, carbon fiber can bridge distant and unconnected graphite particles increasing the overall conductivity of the system. However, the above mentioned authors did not perform experiments with non-conductive particles of similar characteristics. Therefore, the need of a second conductive particle has not been proved in this context.

The above mentioned shows that the effect of a third component on the electrical performance of polymer/CNT composites, forming a hybrid composite material, is not completely understood justifying further investigations. In this article, we analyze how the type of particle can tune the geometrical electrical percolation of polypropylene/CNT composites. In particular, layered-clay, spherical copper metal, and spherical silica particles of different diameters were studied. Our results show that the final conductivity of the hybrid composite mate- rial depends on the particle aspect ratio and that models such as excluded volume and interparticle bridge are not able to explain the whole experimental evidence. The effect of annealing in the melt on the conductivity of the samples was further studied.

\section{Experimental}

A commercial grade polypropylene from Petroquim S.A. (Chile) (PH1310) with a melt flow rate $\left(230^{\circ} \mathrm{C} / 2.16 \mathrm{~kg}\right.$ ) of $13 \mathrm{~g} / 10 \mathrm{~min}$ (Norm ASTM D$1238 / 95$ ) was used as matrix. The multiwalled carbon nanotubes (MWCNT) were kindly supplied by Bayer Material Science AG (Germany) (Baytubes $\mathrm{C} 150 \mathrm{P})$. Based on the datasheet information provided by Bayer, they are characterized by a purity higher than $95 \mathrm{wt} \%$, number of walls between 2 and 15 , an outer mean diameter of $13-16 \mathrm{~nm}$, an inner mean diameter of $4 \mathrm{~nm}$, length between 1 and $>10 \mu \mathrm{m}$, and a bulk density around $150 \mathrm{~kg} / \mathrm{m}^{3}$. The montmorillonite clay filler is a Cloisite 15A from Southern Clay Product Inc. (USA) with a cation exchange capacity (CEC) of $125[\mathrm{meq} / 100 \mathrm{~g}$ of clay]. The copper nanoparticles (CuNP) with an average particle size of $5 \mathrm{~nm}$ as measured by high resolution transmission electron microscopy (TEM) were supplied by Versus Co. Ltd. (Chile).

Spherical silica nanoparticles of $100 \mathrm{~nm}$ were synthesized by the sol-gel method using a two-stage mixed semi-batch method, as previously reported [9]. In the first stage, Solutions A and B were prepared. For Solution A, $0.5 \mathrm{M}$ of thetraethylortosilicate (TEOS) was dissolved in $22 \mathrm{~mL}$ of ethanol whereas in Solution B, $0.2 \mathrm{M}$ of aqueous ammonia solution was added to $23 \mathrm{~mL}$ of ethanol and $2 \mathrm{~mL}$ of water. Solution B was then added dropwise to Solution A. The resulting mixture was allowed to react for 60 minutes at $40^{\circ} \mathrm{C}$ under a $\mathrm{N}_{2}$ atmosphere. In the second stage, Solutions A and B were again prepared and added to the reactor containing the solution and the particles prepared in the first stage. The solutions were separated by centrifugation (10.000 rpm, 20 minutes) and washed three times with ethanol. For the $10 \mathrm{~nm}$ spherical particles a similar procedure was carried out but adding $23 \mathrm{~mL}$ of ethanol and $55 \mathrm{~mL}$ of TEOS in Solution A and $23.5 \mathrm{~mL}$ of ethanol, $54 \mathrm{~mL}$ of water and $2.2 \mathrm{~mL}$ of ammonia for Solution B. The nanoparticles were then calcined for 4 hours at $450^{\circ} \mathrm{C}$ and dried for 6 hours at $100^{\circ} \mathrm{C}$. 
The composites and the master batch for clay filler were prepared by using a Brabender Plasticorder (Brabender, Germnay) internal mixer at $190^{\circ} \mathrm{C}$ and a speed of $110 \mathrm{rpm}$. For PP/CNT composites, predetermined amounts of the nanofiller, antioxidant and neat polymer were mixed under nitrogen atmosphere in order to obtain the composites with filler content ranges from 0.5 to 4 vol\%. First, a half of polymer pellets (around $1 \mathrm{~cm}$ size) with the antioxidant (powder) were added to the mixer operated at $75 \mathrm{rpm}$. After 3 minutes approximately for melt the polymer, the filler was added during 2 minutes. Finally, the rest of polymer pellets was added and the speed of the mixer was raised a $110 \mathrm{rpm}$ for 10 minutes. Therefore, the total mixing time was around 16 minutes. For the hybrid materials, first the polymer was added to the mixer followed by the proper amount of CNT as above explained and afterwards, the desired amount of the second nanoparticle to prepare hybrid materials was added. In this case, the total time for the mixing was around 20 minutes. To analyse the effect of mixing time on the electrical behaviour of PP/CNT composites, several times were tested in the mixer but not effect was detected in the electrical behaviour. For PP/ $\mathrm{CNT} /$ clay hybrid composites a commercial polypropylene grafted with maleic anhydride (Polybond 3200 , Chemtura, USA) was further used as compatibilizer. In this case, a master batch containing a mixture of silica layered particles and the compatibilizer with a weight ratio of 1:3 was prepared under the same conditions as explained above.
Transmission electron microscopy (TEM) images were made in a Philips (The Nteherlands) model CM 100 at $80 \mathrm{kV}$. Ultrathin sections of about $70 \mathrm{~nm}$ were obtained by cutting the samples with an Ultracut Reichert-Jung (Germany) microtome equipped with a Diatome diamond knife. For the electric resistivity, different meghommeters (Megger BM11 with a highest voltage of $1200 \mathrm{~V}$ and AEMC 1060 with a highest voltage of $5000 \mathrm{~V}$ ) were used depending on the conductivity of the samples. With this set-up the standard two-points method was used. These values were compared with those coming from a commercial four-points methods (Jandel RM3-AR, Multiheight Probe'), and not relevant differences were detected. For each electrical value displayed in this contribution, at least three samples were prepared and four measurements for each one were carried out. In general, differences around one order of magnitude were detected in the non-percolated samples having low conductivity values $\left(\sim 10^{-9} \mathrm{~S} / \mathrm{cm}\right)$. For percolated samples otherwise the experimental error for conductivities was less than $50 \%$. For annealing tests, the samples were putted in a hot-press at $190^{\circ} \mathrm{C}$ for 30 minutes without pressure. For these tests, samples of $50 \times 10 \mathrm{~mm}^{2}$ and a thickness of $1 \mathrm{~mm}$ were used.

\section{Results and discussion}

\subsection{Polypropylene/CNT composites}

Figure 1a displays a representative image of a composite of polypropylene with $2.4 \mathrm{vol} \%$ of multi walled carbon nanotubes (CNT). The CNT aggre-

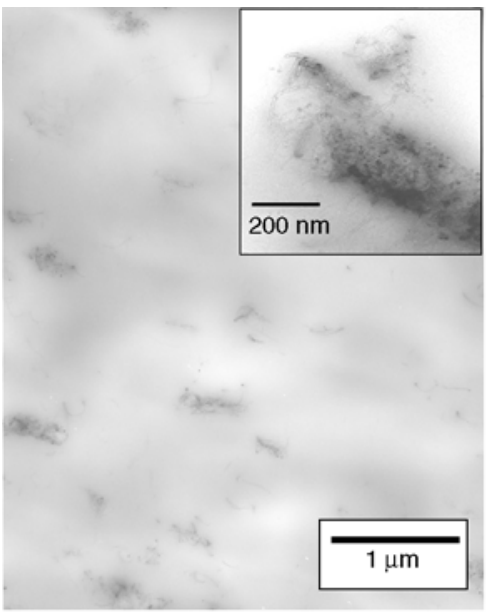

a)

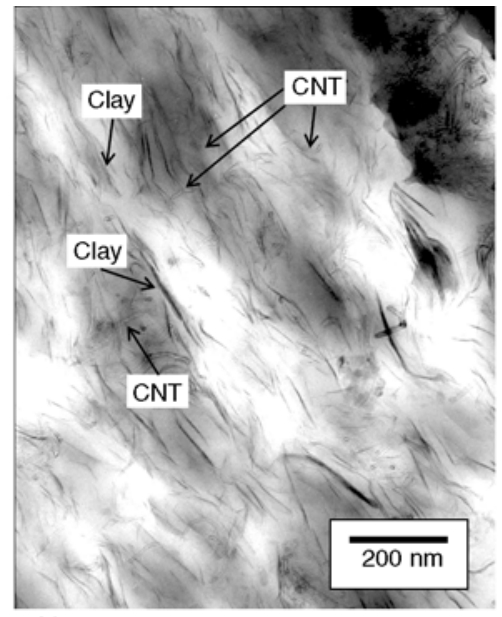

b)

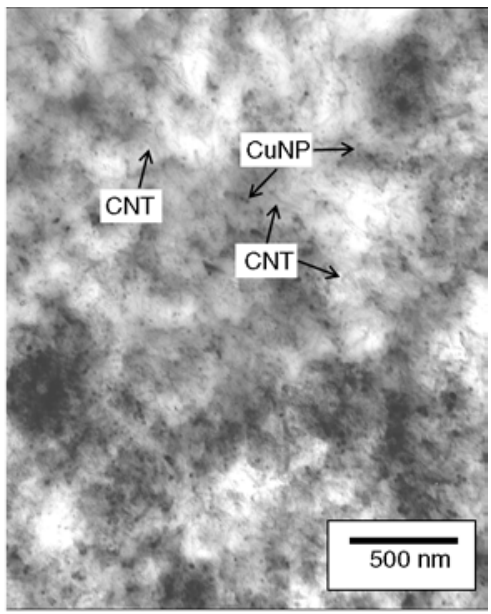

c)

Figure 1. Representative TEM images of some samples studied: a) polypropylene/CNT composites with 2.4 vol $\%$ of filler; b) polypropylene with $2.4 \mathrm{vol} \%$ of CNT and $5.5 \mathrm{vol} \%$ of clay; and c) polypropylene with $2.4 \mathrm{vol} \%$ of CNT and $10 \mathrm{vol} \%$ of copper nanoparticles (CuNP) 


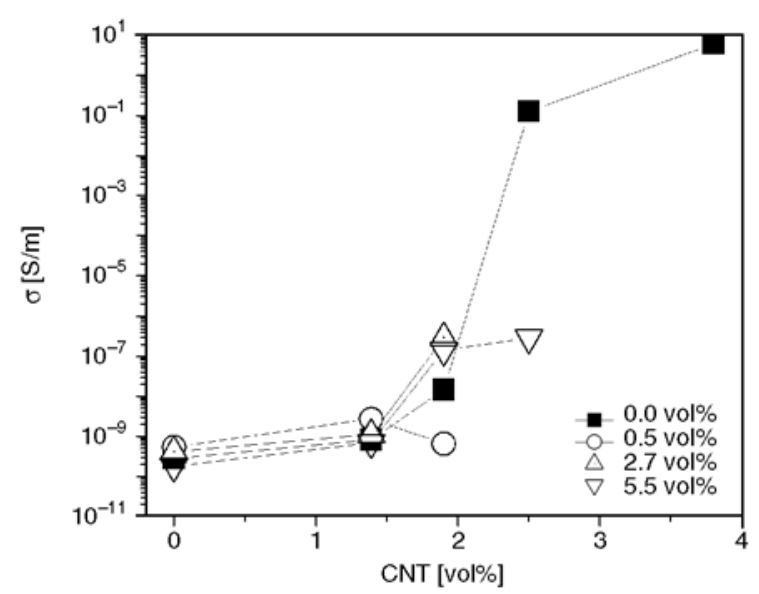

Figure 2. Effect of clay particles on the electrical conductivity of polypropylene/CNT composites

gate forming secondary structures with sizes around $400 \mathrm{~nm}$ that are well distributed into the polymer matrix. Together with these agglomerates, isolated CNT can also be found as reported previously [10, 11]. In the in-set of Figure 1a, there is an image at larger magnification showing the characteristic morphology of isolated CNT by regular resolution TEM. The lateral wall of the tubes are clearly observed because the higher electron density (the (002) lattice image) whereas the top/bottom part of the tubes cannot be detected appearing as transparent. Although it is not observed by our TEM images, the secondary structures can be continuously interconnected through the polymer by means of isolated CNTs forming a network. This hypothesis is confirmed observing Figure 2 where the electrical conductivity is plotted against the CNT concentration. At concentrations higher than 2 vol\% a drastic increase in the conductivity of the composites is displayed because of the presence of a continue conduction path of CNT through the polymer matrix $[1,12]$. Based on this information, a set of hybrid composite materials containing different concentrations of CNT and either copper metal or silica nanoparticles, were prepared and characterized in order to discuss the effect of a second nanoparticle on the electrical behaviour of $\mathrm{PP} / \mathrm{CNT}$ composites.

\subsection{Layered particles}

There are several articles confirming that high aspect ratio particles, such as layered clays, increase the electrical conductivity of $\mathrm{PP} / \mathrm{CNT}$ composites. However, our results show that well dispersed layered fillers do not raise the conductivity as observed in Figure 2. Moreover, with 5.5 vol\% of clay, the layered filler drastically reduces the conductivity of the composites showing a disruption of the CNT network. The restrictions that layered fillers cause on the CNT network can be further observed by TEM images as displayed in Figure 1b. In this figure, clay appears as dense layered particles contrary to CNT where the lateral walls are only observed as discussed previously. Therefore, a reduction in the volume available for CNT dispersion due to the clays is not the only effect of these layered fillers. High aspect ratio clay particles can also hinder the interconnection between CNT acting as a wall between them. In this way, based on our results and those from previous literature, we can say that depending on the concentration of clay and its dispersion, two processes compete having opposite consequences: 1) decrease in the free volume available for CNT dispersion increasing the electrical conductivity, as reported previously; and 2) disruption of the CNT network decreasing the conductivity, as our results support.

\subsection{Conductive spherical particles}

Motivated by the recent results obtained by Liang et al. [8] and Balik and coworkers [2,3] showing that a conductive second nanoparticle can increase the electrical conductivity of polymer/CNT composites, we added copper metal nanoparticles (CuNP) to $\mathrm{PP} / \mathrm{CNT}$ composites. Figure $1 \mathrm{c}$ show a representative image of this hybrid composite material where the black-spot represent the CuNP. If the dispersion of these CuNP in the hybrid composite material is compared with that from PP/CuNP composite [13], a drastic decrease in the agglomeration level is observed when CNT are presented. In particular, Figure 1c display well dispersed individual CuNP instead of agglomerated structure as in $\mathrm{PP} / \mathrm{CuNP}$ composites [13]. Our results could be explained based on the conclusion of Liu et al. [4] regarding the larger affinity of CNT to clay particles improving its dispersion. In this way, CuNP could have larger affinity to CNT than with PP producing a better dispersion of the inorganic filler in the hybrid composite material. The latter is supported by the recent publications about in-situ synthesis of CuNP onto CNT $[14,15]$.

Figure 3 shows that by adding spherical conductive particles the overall conductivity of the composite 


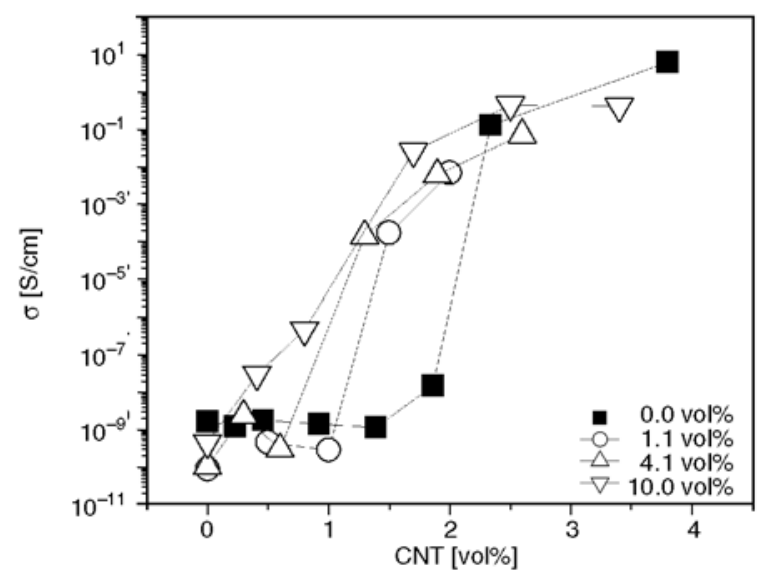

Figure 3. Effect of copper metal nanoparticles on the electrical conductivity of polypropylene/CNT composites

is increased and the percolation threshold is reduced by a factor of two. Moreover, the conductivity depends on the amount of CuNP in the material. An interesting characteristic of these novel hybrid materials is that the electrical conductivity when they are percolated is similar to those obtained from the composites with CNT.

\subsection{Non-conductive spherical particles}

Based on our results and previous articles, metal particles acting as bridge between CNT, or vice versa, is a plausible mechanism to explain the enhanced electrical conductivity in this kind of hybrid composite materials. However, the addition of non-conductive fillers to $\mathrm{PP} / \mathrm{CNT}$ composites as similar as CuNP is the only way to confirm this bridge-theory. In this context, two silica particles of 10 and $100 \mathrm{~nm}$ were synthesized by the sol-gel method. Particles of $10 \mathrm{~nm}$ represent the isolated $\mathrm{CuNP}$ whereas those of $100 \mathrm{~nm}$ are prepared as secondary structures of CuNP in PP matrices are of this order of magnitude in size [13].

Figure 4 shows the results of adding $10 \mathrm{vol} \%$ of non-conductive silica particles to $\mathrm{PP} / \mathrm{CNT}$ composites where it is also displayed the results from hybrid composite materials prepared with CuNP. Results from Figure 4 clearly show that non-conductive fillers are able to increase drastically the conductivity of the composite reducing the percolation threshold by a factor of two. The effect of these non-conductive filler is size-dependent as $10 \mathrm{~nm}$ particles render larger enhancements to the electrical conductivity than $100 \mathrm{~nm}$ particles. Noteworthy,

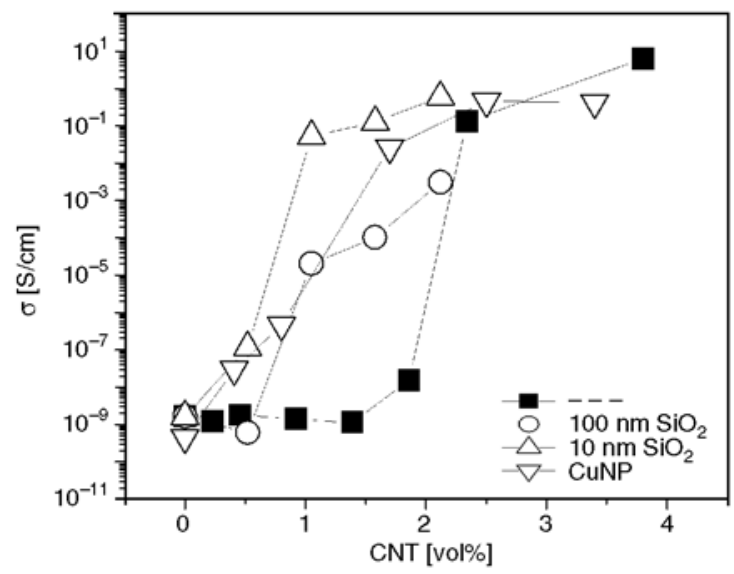

Figure 4. Effect of adding 10 vol\% of different nanoparticles to the electrical conductivity of polypropylene/CNT composites

the improvement in conduction by these fillers is similar to that caused by CuNP ruling out the hypothesis that conductive particles can act as a bridge between CNT or vice versa. A plausible mechanism explaining these findings is the increased effective concentration of CNT in the polymer matrix as spherical particles can reduce the available volume for filler dispersion as discussed previously for other fillers.

Therefore, based on the information displayed in Figure 2, 3 and 4, we can conclude that the effect of a second nanoparticle on the electrical conductivity of PP/CNT composites can be explained by two complementary and competitive mechanisms: 1) the excluded volume theory and 2) the disruption of the CNT network by the filler. Layered clay fillers disrupt the geometrical percolation of the composite reducing the conductivity whereas spherical particles reduce the free volume for CNT dispersion increasing the effective concentration and the electrical conductivity of the composite.

\subsection{Annealing effect}

It is well known that the conductivity of polymer/ CNT composites can be increased by annealing at temperatures higher than the melting temperature of the matrix $[16,17]$. These changes in conductivity are related with the non-equilibrium state of $\mathrm{CNT}$ in high viscous matrices under these quiescent conditions [18] driven by either the entropic forces of selforganization [19] or the high attractive interparticle potential [20-22] overcoming its limited Brownian motion. These processes together with the filler re- 


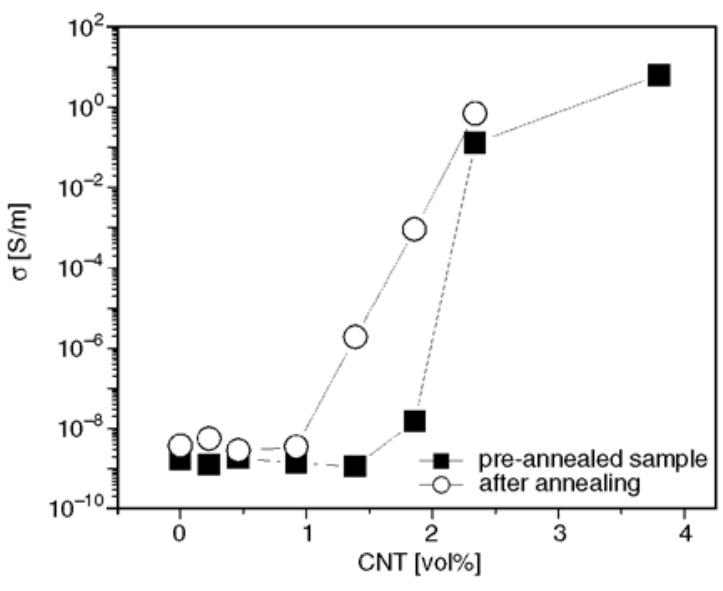

Figure 5. Effect of annealing ( 30 minutes at $190^{\circ} \mathrm{C}$ ) on the electrical conductivity of polypropylene/CNT composites

arrangements due to relaxation of polymer chains at high temperatures, can form conductive agglomerates from the initially dispersed CNT [16, 22-24]. Independent of the mechanism, under annealing the mean distance between CNT, or its clusters, decreases causing enhanced conductivities [17]. This hypothesis regarding agglomeration of CNT under annealing, although at higher pressure, has been recently observed by TEM images [11].

Figure 5 displays the effect of annealing on the electrical behaviour of PP/CNT composites. As discussed above, at high temperatures the composites increase the electrical conductivity due to a rearrangement of CNT decreasing its mean distance [17]. Although this tendency is well known in polymer/CNT composites, the effect of a second nanoparticle on this behaviour has not been reported. Regarding layered particles as second filler, our results (not shown) indicate that there is no a significant effect of annealing on the conductivities of $\mathrm{PP} / \mathrm{CNT}$ composites and changes in the same range as the experimental error are mainly measured. However, when spherical particles are used, more interesting results are observed as the effect will depend on the particle size and its concentration. Figure 6 displays the effect of annealing on the electrical conductivity of $\mathrm{PP} / \mathrm{CNT} / \mathrm{CuNP}$ hybrid composite materials where the data from nonannealed samples are also displayed for comparison. Similar to PP/CNT composites, annealing process is able to increase the conductivity of the samples but the final effect depends on the amount of the second filler. At low CuNP concentration, a softly increase

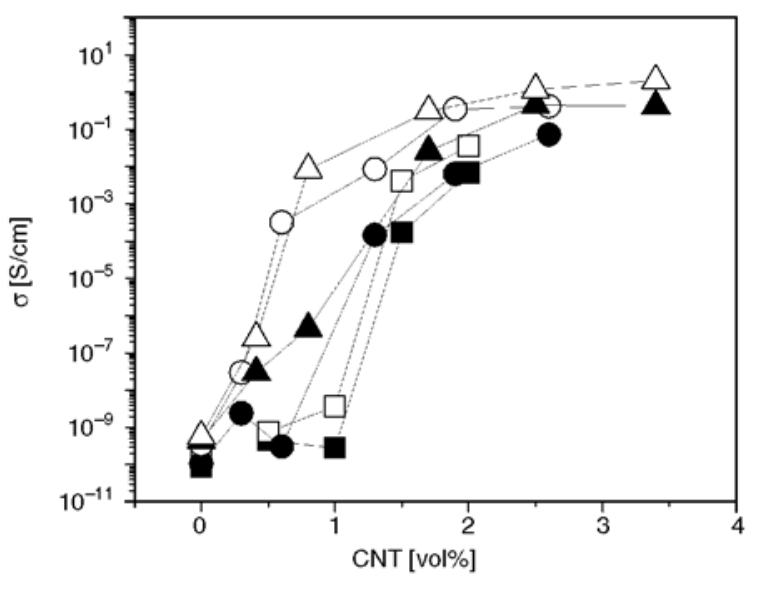

Figure 6. Effect of annealing $\left(30\right.$ minutes at $\left.190^{\circ} \mathrm{C}\right)$ on the electrical conductivity of polypropylene/CNT/ copper nanoparticle (CuNP) hybrid composite materials. Square: 1,1 vol\% of CuNP; circle: 4,1 vol\% of CuNP; and triangle: 10 vol $\%$ of CuNP. Black symbols represent pre-annealed samples whereas white symbols represent the annealed samples.

(in the range of the experimental error) is mainly observed independent of the amount of CNT. These increases are lower than those obtained from PP/ CNT meaning that similar to layered fillers, low CuNP content reduces or hinders the re-arrangement processes of CNT.

The effect of annealing in PP/CNT/silica nanoparticle hybrid composites is displayed in Figure 7. Our results indicate that the annealing effect depends on the size of the second filler as samples with particles of $10 \mathrm{~nm}$ do not show any effect whereas sam-

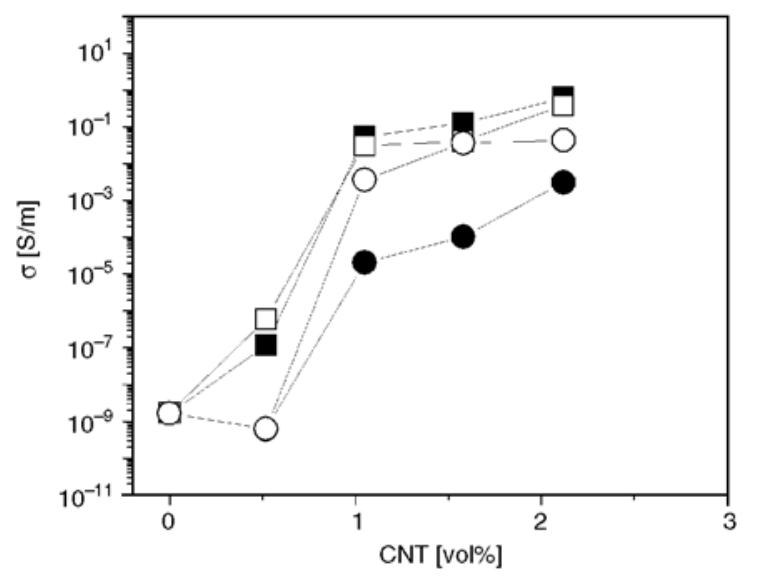

Figure 7. Effect of annealing (30 minutes at $190^{\circ} \mathrm{C}$ ) on the electrical conductivity of polypropylene/CNT hybrid composite materials with $10 \mathrm{vol} \%$ of silica nanoparticles. Square: $10 \mathrm{~nm}$ particles and circle: $100 \mathrm{~nm}$ particles. Black symbols represent preannealed samples whereas white symbols represent the annealed samples. 
ples with particles of $100 \mathrm{~nm}$ display strong increases in conductivities. Regarding samples with $10 \mathrm{~nm}$ particles, there are two plausible theories about the lack of annealing effect: 1) nanoparticles hinder the re-arrangement of CNT similar to that discussed in the case of CuNP and clay; and 2) the presence of a second nanoparticle already improves the conductivity of the pre-annealed sample and therefore to increase even more the conductivity is not possible. The latter is based on the fact that these hybrid composite materials have the highest conductivities when compared with the other samples. On the other hand, the difference observed between samples with CuNP and samples with silica nanoparticles of $10 \mathrm{~nm}$ can be related with the agglomeration processes in the former system as reported previously [13].

\section{Conclusions}

The effect of a second nanoparticle on the electrical behaviour of polypropylene/CNT materials was studied. Our results show that the final conductivity of the hybrid composite depends on the characteristic of the second filler. In particular, layered fillers disrupt the geometrical percolation decreasing the conductivity of the sample whereas nano-spheres could reduce the available volume for CNT dispersion decreasing the electrical percolation threshold. Furthermore, conductive CuNP and non-conductive silica nanoparticles have the same effect ruling out the bridge theory as reported previously. Regarding annealing effect, polypropylene/CNT composites and hybrid composite materials with high amount of the second filler show relevant increases in the electrical conductivity with time. However, layered fillers and low concentration of spherical nanoparticles hinder the re-arrangement of CNT avoiding the annealing effect at high temperatures.

\section{Acknowledgements}

The authors gratefully acknowledge the financial support of CONICYT, projects FONDECYT 1110078. It is also expressed the thanks to Dr. W. Sierralta for the TEM images and to Dr. R. Quijada for the support during this research.

\section{References}

[1] Moniruzzaman M., Winey K. I.: Polymer nanocomposites containing carbon nanotubes. Macromolecules, 39, 5194-5205 (2006). DOI: $10.1021 / \mathrm{ma} 060733 \mathrm{p}$
[2] Thongruang W., Spontak R. J., Balik C. M.: Bridged double percolation in conductive polymer composites: An electrical conductivity, morphology and mechanical property study. Polymer, 43, 3717-3725 (2002). DOI: $10.1016 / \mathrm{S} 0032-3861(02) 00180-5$

[3] Thongruang W., Spontak R. J., Balik C. M.: Correlated electrical conductivity and mechanical property analysis of high-density polyethylene filled with graphite and carbon fiber. Polymer, 43, 2279-2286 (2002). DOI: $10.1016 / \mathrm{S} 0032-3861(02) 00043-5$

[4] Liu L., Grunlan J. C.: Clay assisted dispersion of carbon nanotubes in conductive epoxy nanocomposites. Advanced Functional Materials, 17, 2343-2348 (2007). DOI: $10.1002 / \mathrm{adfm} .200600785$

[5] Bao H-D., Guo Z-X., Yu J.: Effect of electrically inert particulate filler on electrical resistivity of polymer/ multi-walled carbon nanotube composites. Polymer, 49, 3826-3831 (2008).

DOI: $10.1016 /$ j.polymer.2008.06.024

[6] Kotaki M., Wang K., Toh M. L., Chen L., Wong S. Y., He C.: Electrically conductive epoxy/clay/vapor grown carbon fiber hybrids. Macromolecules, 39, 908-911 (2006).

DOI: $10.1021 / \mathrm{ma} 0522561$

[7] Bao S. P., Liang G. D., Tjong S. C.: Positive temperature coefficient effect of polypropylene/carbon nanotube/montmorillonite hybrid nanocomposites. IEEE Transactions Nanotechnology, 8, 729-736 (2009). DOI: $10.1109 /$ TNANO.2009.2023650

[8] Liang G. D., Bao S. P., Tjong S. C.: Microstructure and properties of polypropylene composites filled with silver and carbon nanotube nanoparticles prepared by melt-compounding. Materials Science and Engineering B, 142, 55-61 (2007).

DOI: $10.1016 /$ j.mseb.2007.06.028

[9] Palza H., Vera J., Wilhelm M., Zapata P.: Spherulite growth rate in polypropylene/silica nanoparticle composites: Effect of particle morphology and compatibilizer. Macromolecular Materials and Engineering, 296, 744-751 (2011).

DOI: $10.1002 /$ mame.201000405

[10] Hu G., Zhao C., Zhang S., Yang M., Wang Z.: Low percolation thresholds of electrical conductivity and rheology in poly(ethylene terephthalate) through the networks of multi-walled carbon nanotubes. Polymer, 47, 480-486 (2006).

DOI: $10.1016 /$ j.polymer.2005.11.028

[11] Alig I., Skipa T., Lellinger D., Pötschke P.: Destruction and formation of a carbon nanotube network in polymer melts: Rheology and conductivity spectroscopy. Polymer, 49, 3524-3531 (2008). DOI: 10.1016/j.polymer.2008.05.037

[12] Alig I., Lellinger D., Dudkin S. M., Pötschke P.: Conductivity spectroscopy on melt processed polypropylene-multiwalled carbon nanotube composites: Recovery after shear and crystallization. Polymer, 48, 1020-1029 (2007).

DOI: $10.1016 /$ j.polymer.2006.12.035 
[13] Delgado K., Quijada R., Palma R., Palza H.: Polypropylene with embedded copper metal or copper oxide nanoparticles as a novel plastic antimicrobial agent. Letters in Applied Microbiology, 53, 50-54 (2011). DOI: 10.1111/j.1472-765X.2011.03069.X

[14] Wang P., Huang B., Wei J., Qin X., Yao S., Zhang Q.: Preparation of $\mathrm{Cu}$ nanoparticles on carbon nanotubes by solution infusion method and calcining in ambient atmosphere. Materials Letters, 61, 5255-5257 (2007). DOI: $10.1016 /$ j.matlet.2007.04.041

[15] Wu H-Q., Wei X-W., Shao M-W., Gu J-S., Qu M-Z.: Synthesis of copper oxide nanoparticles using carbon nanotubes as templates. Chemical Physics Letters, 364, 152-156 (2002).

DOI: $10.1016 / \mathrm{S} 0009-2614(02) 01301-5$

[16] Cipriano B. H., Kota A. K., Gershon A. L., Laskowski C. J., Kashiwagi T., Bruck H. A., Raghavan S. R.: Conductivity enhancement of carbon nanotube and nanofiber-based polymer nanocomposites by melt annealing. Polymer, 49, 4846-4851 (2008). DOI: 10.1016/j.polymer.2008.08.057

[17] Palza H., Kappes M., Hennrich F., Wilhelm M.: Morphological changes of carbon nanotubes in polyethylene matrices under oscillatory tests as determined by dielectrical measurements. Composites Science and Technology, 71, 1361-1366 (2011).

DOI: $10.1016 /$ j.compscitech.2011.05.010

[18] Obrzut J., Douglas F. J., Kharchenko S. B., Migler K. B.: Shear-induced conductor-insulator transition in melt-mixed polypropylene-carbon nanotube dispersions. Physical Review B, 76, 195420/1-195420/9 (2007).

DOI: 10.1103/PhysRevB.76.195420
[19] Skipa T., Lellinger D., Saphiannikova M., Alig I.: Shear-stimulated formation of multi-wall carbon nanotube networks in polymer melts. Physica Status Solidi B, 246, 2453-2456 (2009).

DOI: $10.1002 / \mathrm{pssb} .200982265$

[20] Kharchenko S. B., Douglas J. F., Obrzut J., Grulke E. A., Migler K. B.: Flow-induced properties of nanotube-filled polymer materials. Nature Materials, 3, 564-568 (2004).

DOI: $10.1038 /$ nmat 1183

[21] Hobbie E. K., Fry D. J.: Nonequilibrium phase diagram of sticky nanotube suspensions. Physical Review Letters, 97, 036101/1-036101/4 (2006). DOI: 10.1103/PhysRevLett.97.036101

[22] Huang Y. Y., Ahir S. V., Terentjev E. M.: Dispersion rheology of carbon nanotubes in a polymer matrix. Physical Review B, 73, 125422/1-125422/9 (2006). DOI: $10.1103 /$ PhysRevB.73.125422

[23] Skipa T., Lellinger D., Böhm W., Saphiannikova M., Alig I.: Influence of shear deformation on carbon nanotube networks in polycarbonate melts: Interplay between build-up and destruction of agglomerates. Polymer, 51, 201-210 (2010).

DOI: 10.1016/j.polymer.2009.11.047

[24] Pujari S., Rahatekar S. S., Gilman J. W., Koziol K. K., Windle A. H., Burghardt W. R.: Orientation dynamics in multiwalled carbon nanotube dispersions under shear flow. Journal of Chemistry Physics, 130, 214903/1214903/9 (2009).

DOI: $10.1063 / 1.3139446$ 